University of Nebraska - Lincoln

DigitalCommons@University of Nebraska - Lincoln

Faculty Publications from the Department of Electrical \& Computer Engineering, Department Electrical and Computer Engineering

2-26-1997

\title{
High-bandwidth attitude jitter determination for pointing and tracking systems
}

Marcelo C. Algrain

Follow this and additional works at: https://digitalcommons.unl.edu/electricalengineeringfacpub

Part of the Computer Engineering Commons, and the Electrical and Computer Engineering Commons

This Article is brought to you for free and open access by the Electrical \& Computer Engineering, Department of at DigitalCommons@University of Nebraska - Lincoln. It has been accepted for inclusion in Faculty Publications from the Department of Electrical and Computer Engineering by an authorized administrator of DigitalCommons@University of Nebraska - Lincoln. 


\section{High-bandwidth attitude jitter determination for pointing and tracking systems}

\author{
Marcelo C. Algrain, MEMBER SPIE \\ University of Nebraska-Lincoln \\ Department of Electrical Engineering \\ and \\ Center for Electro-Optics \\ Lincoln, Nebraska 68588-0511 \\ E-mail: malgrain@unlinfo.unl.edu
}

\begin{abstract}
For many reasons smaller spacecraft are becoming more appealing. Because of their lower inertias, these spacecraft are more sensitive to disturbances and likely to have more attitude jitter than the larger units. These jitter levels are unacceptable for some scientific instruments and need to be compensated. In the case of line-of-sight type instruments, the attitude jitter can be mitigated by incorporating a fast steering mirror into the system. To take full advantage of these devices, the spacecraft attitude must be measured at sufficiently high bandwidth, well beyond what is commonly provided by inertial reference units. Various ways to obtain higher bandwidth attitude measurements for the purpose of jitter control are explored and a practical solution to the problem is proposed. (๑) 1997 Society of Photo-Optical Instrumentation Engineers. [S0091-3286(97)02807-9]
\end{abstract}

Subject terms: sensor fusion; inertial reference unit; accelerometer; angular displacement sensor.

Paper 24066 received June 17, 1996; revised manuscript received Jan. 22, 1997; accepted for publication Feb. 26, 1997.

\section{Introduction}

Spacecraft (S/C) attitude can be measured using combinations of sensors, such as, gyros, global positioning system (GPS) receivers, star-tracker units (STU), sun and/or horizon sensors, etc. This type of sensor arrangement forms what is often referred to as an inertial reference unit (IRU). The gyros provide highly accurate short-term attitude data, but suffer from long-term inaccuracies primarily due to drift errors. To correct them, a Kalman filter is commonly used to estimate the drift based on a well-defined gyro drift error propagation model ${ }^{1,2}$ and measurements from a GPS, STU, or similar type instrument. ${ }^{3,4}$ Accuracies in the range of few arcseconds are quite feasible, but bandwidth is typically limited to less than $10 \mathrm{~Hz}$. This is well under what would be needed for measuring attitude jitter. Expanding the gyro bandwidth, while maintaining comparable accuracy, is likely to be neither a practical nor an economically feasible option for the foreseeable future. Furthermore, there is no single sensor currently available that would provide both the accuracy and the high bandwidth needed for combined precision pointing and jitter control. Therefore, other sensing means must be devised.

The approach selected in this paper fuses measurements from high-bandwidth sensors with those provided by lowbandwidth sensors. This enables us to take advantage of the high accuracy of an IRU attitude measurement type at the low end of the frequency spectrum, while incorporating high-frequency data required for jitter control. Among the likely candidates for high-bandwidth sensors are angular displacement sensors (ADSs), accelerometers, and quartz rate sensors. The developments in this paper are applicable to all these types of sensors, but they are tailored to the ADS device to keep the presentation focused.

In short, the purpose of this paper is to provide a practical way for producing accurate high-bandwidth measure- ments of attitude jitter in small spacecraft. The goal is to extend the measurement bandwidth over a range of several hundred hertz. The particulars of the developments are presented in the sections that follow.

\section{High-Bandwidth Attitude Determination}

It is known from 3-D rotational dynamics, that S/C attitude (in body coordinates) can be represented as the matrix $\mathbf{C}$, which can be computed ${ }^{5}$ according to Eq. (1), where $\omega^{\times}$ represents the cross-product operation applied to the angular velocity vector $\boldsymbol{\omega}=\left[\omega_{X} \omega_{Y} \omega_{Z}\right]^{T}$.

$$
\begin{aligned}
\dot{\mathbf{C}}=-\boldsymbol{\omega}^{\times} \mathbf{C}= & -\left[\begin{array}{ccc}
0 & -\omega_{Z} & \omega_{Y} \\
\omega_{Z} & 0 & -\omega_{X} \\
-\omega_{Y} & \omega_{X} & 0
\end{array}\right] \\
& \times\left[\begin{array}{ccc}
C_{11} & C_{12} & C_{13} \\
C_{21} & C_{22} & C_{23} \\
C_{31} & C_{32} & C_{33}
\end{array}\right] .
\end{aligned}
$$

For sufficiently small time increments, the previous equation can be accurately approximated using a first-order difference equation (more precise approximations can also be used if desired). Then, the attitude matrix calculation can be expressed as

$\mathbf{C}(n)=\left[\mathbf{I}-\boldsymbol{\omega}^{\times}(n) T\right] \mathbf{C}(n-1)$,

where $\mathbf{I}$ is a $3 \times 3$ identity matrix, $T$ is the incremental time step (sampling period), and $n$ is the time step index. For simplicity, it is assumed that the S/C frame is initially aligned with a fixed inertial reference frame, and that the initial S/C attitude matrix $(\mathbf{C}$ at $t=0)$ is the identity matrix 
(positional reference). The attitude angles can be determined from the attitude matrix by adopting a suitable attitude convention (Euler's angles). ${ }^{6}$ This arrangement is capable of resolving pointing accuracies of the order of few arcseconds, especially when measurements of $\omega$ are enhanced by a Kalman filter, but the bandwidth is limited to few hertz. Extending the bandwidth would require additional sensors capable of detecting the high-frequency motion components. This approach is presented in the remainder of this section.

A variety of sensors can be used to measure angular jitter. Among them are accelerometers, quartz gyros, and ADSs. The bandwidth on any of these sensors is at least one order of magnitude above that of a typical IRU, and can be as high as several kilohertz. In the case of quartz gyros, or the like, the sensors directly measure angular velocity; thus, the term $\omega^{\times}$in the attitude calculation [see Eq. (2)] can be obtained directly from measurements. Conversely, the ADS device measures angular displacements; thus, angular velocities can be obtained as pseudo-rates of the angular displacement data, as follows:

$\boldsymbol{\omega}(n) \cong \frac{\boldsymbol{\theta}(n)-\boldsymbol{\theta}(n-1)}{T}$,

where $\boldsymbol{\theta}$ represents the angular displacement. Using pseudo-rates has the drawback that the accuracy of the attitude calculations are more susceptible to sensor noise. However, since sensor noise for a device like the ADS is very low, ${ }^{7}$ using pseudo-rates would be a generally acceptable practice. Then, when ADS measurements are used, the attitude calculation of Eq. (2) can be expressed in terms of Eq. (3) as follows:

$\mathbf{C}(n) \cong\left[\mathbf{I}-\boldsymbol{\theta}^{\times}(n)+\boldsymbol{\theta}^{\times}(n-1)\right] \mathbf{C}(n-1)$,

where $\boldsymbol{\theta}^{\times}$results from applying the cross-product operator to the angular displacement vector $\boldsymbol{\theta}=\left[\theta_{X} \theta_{Y} \theta_{Z}\right]^{T}$.

The type of sensor that offers the highest bandwidth capabilities is a linear accelerometer. Recent technological developments have led to a new generation of low-power solid state devices that are smaller, lighter, more accurate, and considerably less expensive than their predecessors. ${ }^{8}$ These devices make measuring angular motion with linear accelerometers a cost-effective alternative to using traditional angular sensors. To illustrate this fact, consider the general sensor arrangement shown in Fig. 1. Triaxial linear accelerometers are placed at points $A, B, C$, and $D$, providing the following twelve outputs: $A_{A}^{X}, A_{A}^{Y}, A_{A}^{Z}, A_{B}^{X}$, $A_{B}^{Y}, A_{B}^{Z}, A_{C}^{X}, A_{C}^{Y}, A_{C}^{Z}, A_{D}^{X}, A_{D}^{Y}$, and $A_{D}^{Z}$, where $A_{D}^{Z}$ is the acceleration at point $D$ in the $Z$ direction, and likewise for the other outputs. Also, since the accelerometer relative positions $R_{B / A}, R_{C / A}$, and $R_{D / A}$ are known, all the necessary data is readily available to extract the desired angular motion information, establishing the following relationships between angular and linear accelerations ${ }^{9}$ :

$\dot{\boldsymbol{\omega}}_{X}=\frac{A_{C}^{Z}-A_{A}^{Z}}{2 R_{C / A}}-\frac{A_{D}^{Y}-A_{A}^{Y}}{2 R_{D / A}}=\Delta A_{X}$,

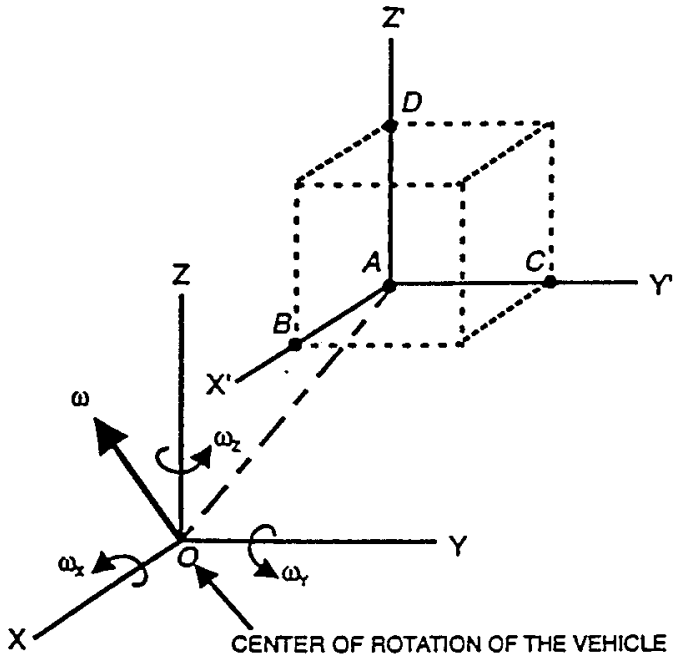

Fig. 1 Linear accelerometer arrangement for angular motion determination.

$\dot{\boldsymbol{\omega}}_{Y}=\frac{A_{D}^{X}-A_{A}^{X}}{2 R_{D / A}}-\frac{A_{B}^{Z}-A_{A}^{Z}}{2 R_{B / A}}=\Delta A_{Y}$,

$\dot{\boldsymbol{\omega}}_{Z}=\frac{A_{B}^{Y}-A_{A}^{Y}}{2 R_{B / A}}-\frac{A_{C}^{X}-A_{A}^{X}}{2 R_{C / A}}=\Delta A_{Z}$.

The previous system of equations demonstrates that nine single-axis linear accelerometers can be used to sense angular accelerations along the three orthogonal axes of rotation. These measurements can be used to calculate vehicle attitude according to Eq. (2). However, since angular accelerations are sensed instead of velocities, a simple first-order approximation can be used to provide the necessary angular velocity term used in Eq. (2), as

$\boldsymbol{\omega}(n) \cong \boldsymbol{\omega}(n-1)+T \Delta A(n)$.

More precise approximations can be used to determine attitude angles. A good choice, because of its good computational characteristics, would be the fourth-order RungeKutta (R-K) algorithm. ${ }^{10}$ Regardless of the numerical method used to integrate Eq. (1), the orthogonality condition $\mathbf{C}^{T} \mathbf{C}=$ I should be periodically examined as a partial check on the accuracy of the integration process.

\section{Sensor Fusion of Low- and High-Bandwidth Measurements}

A common problem associated with high-bandwidth sensors is their inability to accurately register slow-varying motion (low frequencies). For example, consider the case where accelerometers are used to sense angular jitter. Then, rapid motion would yield large accelerometer outputs, while slow motion would generate small acceleration signals. If the accelerometers are selected so that they have enough sensitivity to measure slow motion, they are likely to saturate under rapid motion. Conversely, if they are scaled not to saturate under rapid motion, they would lack the resolution to detect slow motion. This creates problems when trying to resolve attitude angles over a wide range of frequencies. A similar problem is associated with quartz 


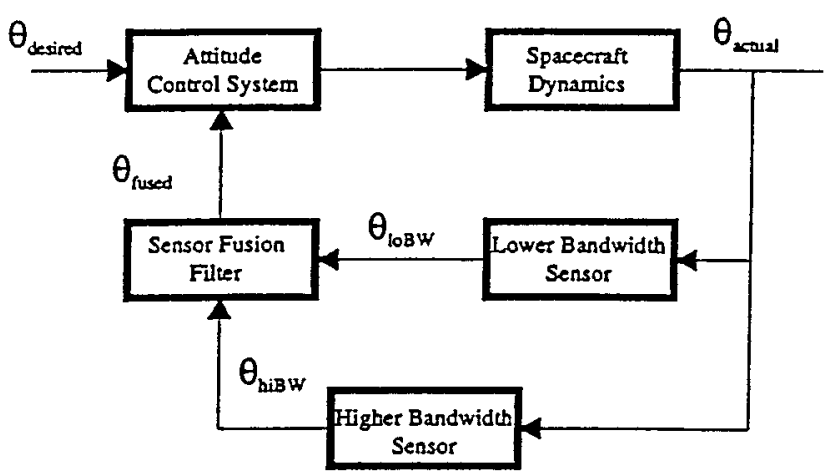

Fig. 2 Composite attitude measurement scheme using low- and high-bandwidth sensors.

gyros and ADS devices since they lack near-dc sensitivity. Therefore, none of these sensors could directly replace the traditional IRU arrangement. However, they can be used in conjunction with an IRU to produce extended-bandwidth measurements of S/C attitude that are accurate over the entire frequency spectrum of interest. Figure 2 shows how a high-bandwidth sensor can be used alongside an IRU and extend its bandwidth. The data provided by the corresponding sensors, $\theta_{\mathrm{loBW}}$ and $\theta_{\mathrm{hiBW}}$, is combined by the sensor fusion filter yielding the extended-bandwidth measurements. The sensor fusion process is described next.

Fusing high-bandwidth measurements with those from a low-bandwidth sensor could be accomplished in a number of ways. One possibility is to use the classic method commonly known as the combining or complementary filter. Its block diagram is shown in Fig. 3. In this approach, outputs from inertial sensors with different frequency response characteristics are added to form a measurement that provides data encompassing the overall frequency range covered by the sensors. Unfortunately, and almost inevitably, there will be some overlap in the frequency response of each sensor (underlap is undesirable since some data would be lost). This creates a condition where some frequencies would be gained more than others. For that reason a filter is introduced to de-weigh the composite measurement. The form of the transfer function for the combining filter is then given in Eq. (7), where $G_{\mathrm{loBw}}$ and $G_{\mathrm{hiBw}}$ are transfer functions of the low-bandwidth inertial unit, and the highbandwidth inertial sensor, respectively:

$G_{\text {fused }}=\frac{1}{G_{\mathrm{loBW}}+G_{\mathrm{hiBW}}}$.
In the case of a specific sensor, knowing its transfer function can be a fair assumption, but the overall transfer function of an inertial reference unit may not be so readily available. It involves gyro dynamics, Kalman filtering, startracker or GPS, etc. This creates uncertainties resulting in inaccurate measurements. Furthermore, the combining filter method relies heavily on the roll-off characteristics of the frequency response for each sensor. This creates significant uncertainty since most sensors show largest variations in this region. Thus, the combining or complementary filter approach would be impractical for many applications.

Another possible approach is to use a Kalman filter to estimate the low-frequency error term associated with the data provided by a high-bandwidth sensor. At first glance, it would seem that the problem is the converse to estimating the drift in a gyro. In other words, a gyro incorporates a low-frequency error-term (random walk/drift) into the measurement, while the high-bandwidth sensor removes the low-frequency motion component from the measurement. That is where the similarities end. This is because there is no apparent propagation model for the low-frequency term in a high-bandwidth measurement (it is simply not there), while the gyro drift propagation model is well known and has been extensively studied. In the absence of a system model, it is conceivable to use an extended Kalman filter to estimate the correction term as a slowly varying parameter. However, this would require a high-order time-variant Kalman filter, which was deemed as impractical for this application.

To overcome the shortcomings of the previous approaches, an alternative method is hereby proposed. In this case, the blending of low- and high-bandwidth measurements is accomplished by using a closed-loop controller to regulate the long-term characteristics of the attitude angles yielded by the high-bandwidth data. In describing this approach, while keeping the discussions focused, consider the case where a low-bandwidth IRU is used in combination with a high-bandwidth ADS device. The reason for this selection is that an IRU is a common choice for measuring attitude, and the ADS has several highly desirable features, such as, low noise characteristics, high accuracy, and large bandwidth. In any case, the methodology is applicable (or easily extendable) to other configurations.

In contrast to the classical approaches previously mentioned, combining and Kalman filtering, the closed-loop controller method does not require a model or transfer function for either the ADS or the IRU. Instead, the lack of dc response on the ADS sensors is overcome by matching

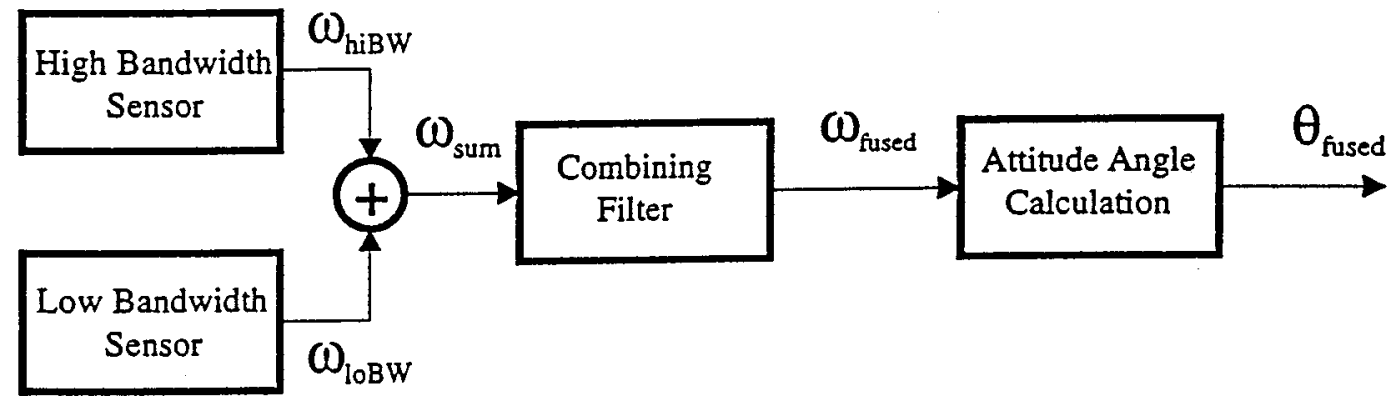

Fig. 3 Combining filter approach to fusion of low- and high-frequency measurements. 
Algrain: High-bandwidth attitude jitter determination ...

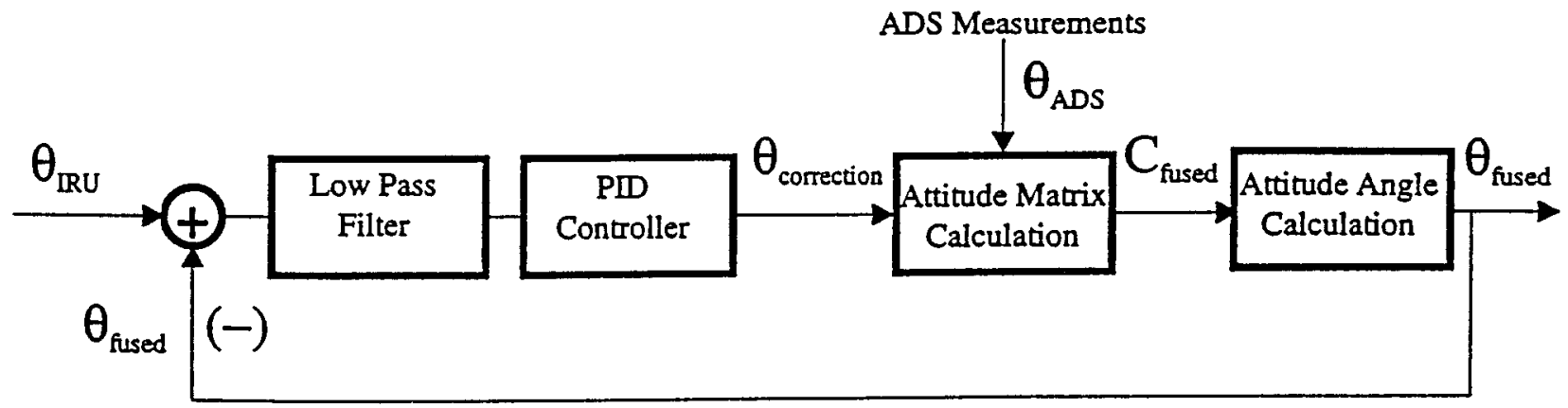

Fig. 4 Closed-loop control of near-dc response.

the long-term characteristics of the S/C attitude measured using ADS outputs to those corresponding to the IRU. This is accomplished using a closed-loop control system. The controller choices are multiple. A good combination is to use a versatile proportional-plus-integral-plus-derivative (PID) controller and a low-pass filter (LPF). Figure 4 shows a block diagram for this approach. Basically, the function of the LPF is to suppress the high-frequency data introduced by the feedback loop, while the PID controller generates the correction term incorporating the low-frequency data not registered by the ADS. When this term is factored in, the attitude calculation based on ADS measurements takes the following form:

$$
\begin{aligned}
{ }_{\text {fused }} \mathbf{C}(n)= & {\left[\mathbf{I}-{ }_{\mathrm{ADS}} \boldsymbol{\theta}^{\times}(n)+{ }_{\mathrm{ADS}} \boldsymbol{\theta}^{\times}(n-1)-{ }_{\text {cor }} \boldsymbol{\theta}^{\times}(n)\right.} \\
& \left.+{ }_{\text {cor }} \boldsymbol{\theta}^{\times}(n-1)\right]_{\text {fused }} \mathbf{C}(n-1),
\end{aligned}
$$

where

$\mathrm{ADS} \boldsymbol{\theta}^{\times}=\left[\begin{array}{ccc}0 & -{ }_{\mathrm{ADS}} \theta_{Z} & \mathrm{ADS} \theta_{Y} \\ \mathrm{ADS} \theta_{Z} & 0 & -{ }_{\mathrm{ADS}} \theta_{X} \\ -{ }_{\mathrm{ADS}} \theta_{Y} & \mathrm{ADS} \theta_{X} & 0\end{array}\right]$

and

$\operatorname{cor} \boldsymbol{\theta}^{\times}=\left[\begin{array}{ccc}0 & -{ }_{c o r} \theta_{Z} & { }_{c o r} \theta_{Y} \\ { }_{\operatorname{cor}} \theta_{Z} & 0 & -{ }_{\operatorname{cor}} \theta_{X} \\ -{ }_{\operatorname{cor}} \theta_{Y} & { }_{\operatorname{cor}} \theta_{X} & 0\end{array}\right]$,

where ${ }_{\mathrm{ADS}} \theta_{X},{ }_{\mathrm{ADS}} \theta_{Y}$, and ${ }_{\mathrm{ADS}} \theta_{Z}$ are the angular displacements sensed along the roll, pitch, and yaw axes, respectively; and ${ }_{\operatorname{cor}} \theta_{X},{ }_{\text {cor }} \theta_{Y}$, and ${ }_{\text {cor }} \theta_{Z}$ are the low-frequency correction terms generated by the closed-loop controller. These correction terms are generated using three separate but identical PID controllers. Each controller is associated with one axis of rotation. The input to a given PID controller is a low-pass-filtered version of the difference between the attitude angle determined by the IRU (for the corresponding axis) and the one obtained from corrected ADS data, as shown below, where $G_{\mathrm{LPF}}$ and $G_{\mathrm{PID}}$ are transfer functions for the LPF and PID controller, respectively:

${ }_{\text {cor }} \theta_{i}=G_{\mathrm{LPF}} G_{\mathrm{PID}}\left({ }_{\mathrm{IRU}} \theta_{i}-{ }_{\text {fused }} \theta_{i}\right) \quad$ with $i=X, Y, Z$.

The PID gains and the LPF bandwidth should be selected such that they would yield a reasonably small band- width for the closed-loop controller. In other words, if the closed-loop controller is too soft, the corrected ADS attitude would not be able to track the IRU attitude. On the other hand, if the closed-loop controller is too stiff, the higher frequency terms would dominate the low-frequency correction term ${ }_{\operatorname{cor}} \theta_{i}$ and not yield the effect sought.

\section{Computer Simulation Results}

A computer simulation was used to assess the performance of the closed-loop-controlled sensor fusion approach for ADS and IRU measurements. To simplify the simulation, the low-bandwidth sensor (IRU) was modeled as a thirdorder Butterworth LPF with a bandwidth of $10 \mathrm{~Hz}$. Its transfer function is given by

$G_{\mathrm{IRU}}=\frac{248050}{s^{3}+125.664 s^{2}+7895.69 s+248050}=G_{\mathrm{loBW}}$.

The model for the ADS was provided by the manufacturer, ${ }^{7}$ and it represents a third-order high-pass filter with passband starting at $2 \mathrm{~Hz}$. Its transfer function is

$G_{\mathrm{ADS}}=\frac{s^{3}}{s^{3}+18.6 s^{2}+158 s+69}=G_{\mathrm{hiBW}}$.

The closed-loop controller accomplishing the high- and low-bandwidth sensor fusion (see Fig. 4) uses a third-order Butterworth LPF. The LPF cutoff frequency is $5 \mathrm{~Hz}$, which is near the geometric center of the cutoff frequencies for the ADS and IRU ( 2 and $10 \mathrm{~Hz}$, respectively). Its transfer function is given by

$G_{\mathrm{LPF}}=\frac{31006.3}{s^{3}+62.8318 s^{2}+1973.92 s+31006.3}$.

The PID controller has gains $K_{P}=0.1$ (proportional), $K_{I}=10$ (integral), $K_{D}=0.01$ (derivative), and the following transfer function:

$G_{\mathrm{PID}}=\frac{K_{D} s^{2}+K_{P} s+K_{I}}{s}$.

All these transfer functions are synthesized digitally. The sampling rate for the IRU is $T_{\text {IRU }}=0.01 \mathrm{~s}$. The sampling rate for the ADS and the closed-loop sensor fusion filter is $T_{\mathrm{ADS}}=0.001 \mathrm{~s}$. 


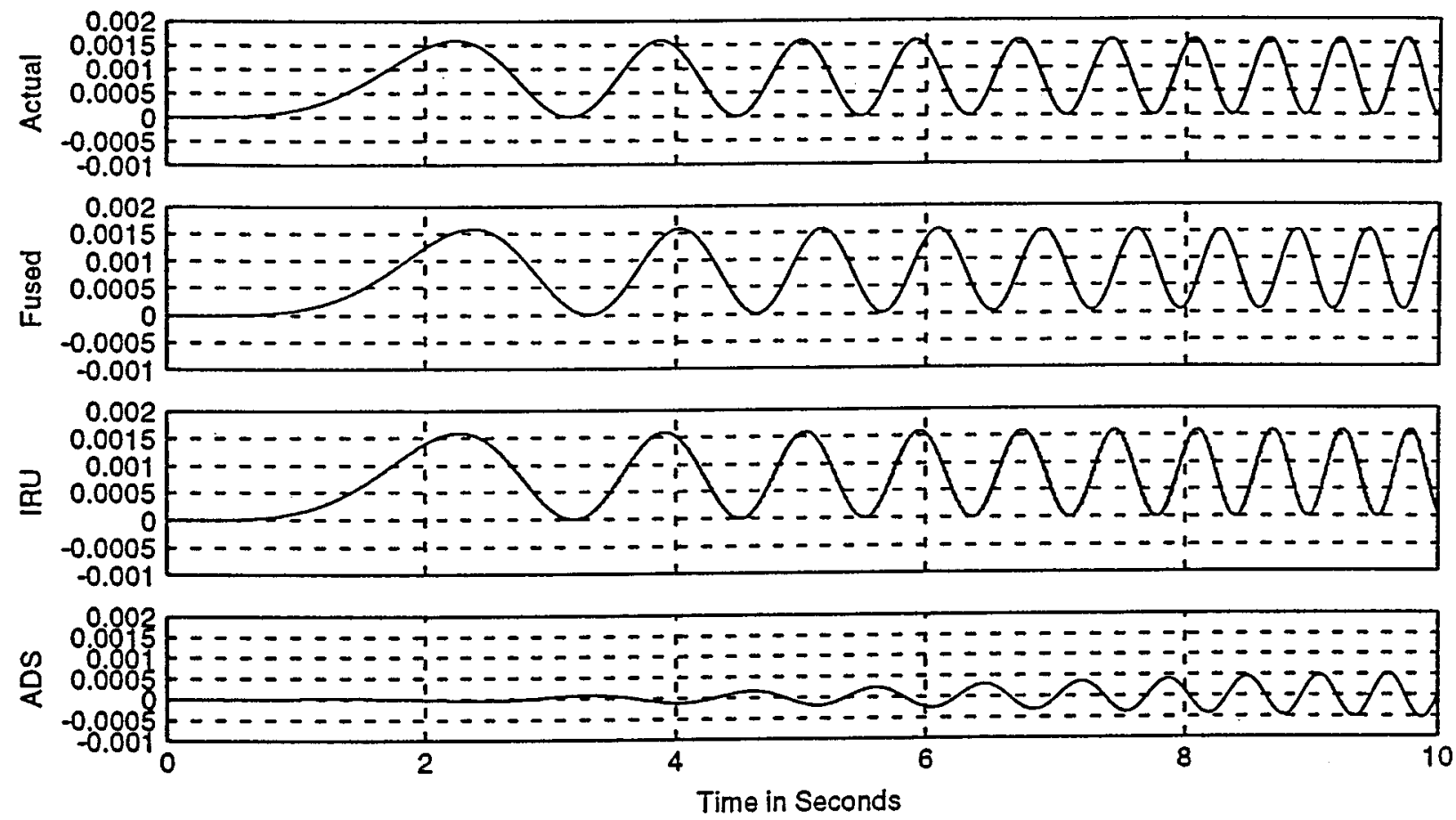

Fig. 5 Attitude angle corresponding to slow sinusoidal frequency sweep (roll axis).

The first simulation test case consists of sensing a sinusoidal motion of increasing frequency (swept sine wave). The actual motion rates along the principal axes of rotation are defined as follows:

Roll: $\quad \boldsymbol{\omega}_{X}=10^{-3} t \sin \left(0.2 \pi t^{2}\right)(\mathrm{rad} / \mathrm{s})$,

Pitch: $\quad \boldsymbol{\omega}_{Y}=10^{-2} t \sin \left(2 \pi t^{2}\right)(\mathrm{rad} / \mathrm{s})$,

Yaw: $\quad \boldsymbol{\omega}_{Z}=10^{-1} t \sin \left(20 \pi t^{2}\right)(\mathrm{rad} / \mathrm{s})$.

Since in this case we are primarily interested in measuring attitude jitter, small angle approximations are applicable, and the S/C attitude angles (in the roll, pitch, and yaw axes) can be extracted from the resulting attitude matrix as follows:

$\boldsymbol{\theta}=f(\mathbf{C})=\left[\theta_{X} \theta_{Y} \theta_{Z}\right]^{T}$,

where

$\theta_{X}=1 / 2\left(C_{23}-C_{32}\right), \quad \Theta_{Y}=1 / 2\left(C_{31}-C_{13}\right)$,

$\theta_{Z}=1 / 2\left(C_{12}-C_{21}\right)$,

and $C_{i j}$ representing the element of the matrix $\mathbf{C}$ in row $i$ and column $j$.

During the 10-s simulation, the roll axis experiences sinusoidal angular rates in the range of 0 to $1 \mathrm{~Hz}$, the pitch axis sees rates from 0 to $10 \mathrm{~Hz}$, and the yaw axis undergoes rates from 0 to $100 \mathrm{~Hz}$. Likewise, the sinusoidal amplitudes range from 0 to $0.01 \mathrm{rad} / \mathrm{s}$ for the roll axis, 0 to $0.1 \mathrm{rad} / \mathrm{s}$ for the pitch axis, and 0 to $1 \mathrm{rad} / \mathrm{s}$ for the yaw axis. Although some of these rates may be considered excessive for an $\mathrm{S} / \mathrm{C}$, they are used here to dramatize the effectiveness of the closed-loop sensor fusion method in detecting attitude angles with comparable magnitudes all across a frequency spectrum of interest.

The simulation results for the roll axis motion are shown in Fig. 5. The test scenario corresponds to sensing slow motion $(0$ to $1 \mathrm{~Hz})$. The upper trace shows the actual angular displacement, the ADS/IRU fused attitude angle is given below it, the attitude angle extracted from lowbandwidth IRU measurement is next, and the attitude angle from high-bandwidth ADS measurements is given on the bottom trace. Notice that the highest frequency component on this motion is below the sensing range for the ADS device, but well within the IRU bandwidth. These plots demonstrate that the correction term introduced by the PID controller is quite effective in incorporating the lowfrequency data provided by the IRU into the ADS measurements (compare the two plots at the top of the figure).

The results for the pitch axis motion corresponding to midrange frequencies $(0$ to $10 \mathrm{~Hz})$ are shown in Fig. 6 . As before, the traces show (from top to bottom) the actual attitude angle, ADS/IRU fused angle, the IRU angle, and the ADS angle, respectively. In this case, the closed-loop sensor fusion approach also blends the data provided by both low- and high-bandwidth sensors successfully. Recall that the ADS passband starts at $2 \mathrm{~Hz}$, and the IRU has a passband ending at $10 \mathrm{~Hz}$.

The simulation results for the fast motion exerted along the yaw axis are shown* in Fig. 7. In this case, the sinusoidal motion is swept in frequency from 0 to $100 \mathrm{~Hz}$ within $10 \mathrm{~s}$. After approximately $1 \mathrm{~s}$, the accuracy of atti-

\footnotetext{
*Due to the high frequencies involved, and the compressed time scale, the time plots in Fig. 7 quickly lose resolution. However, the important information for this part of the analysis is conveyed by the envelop of the data. The gain data at various frequencies is later presented in Fig. 10.
} 


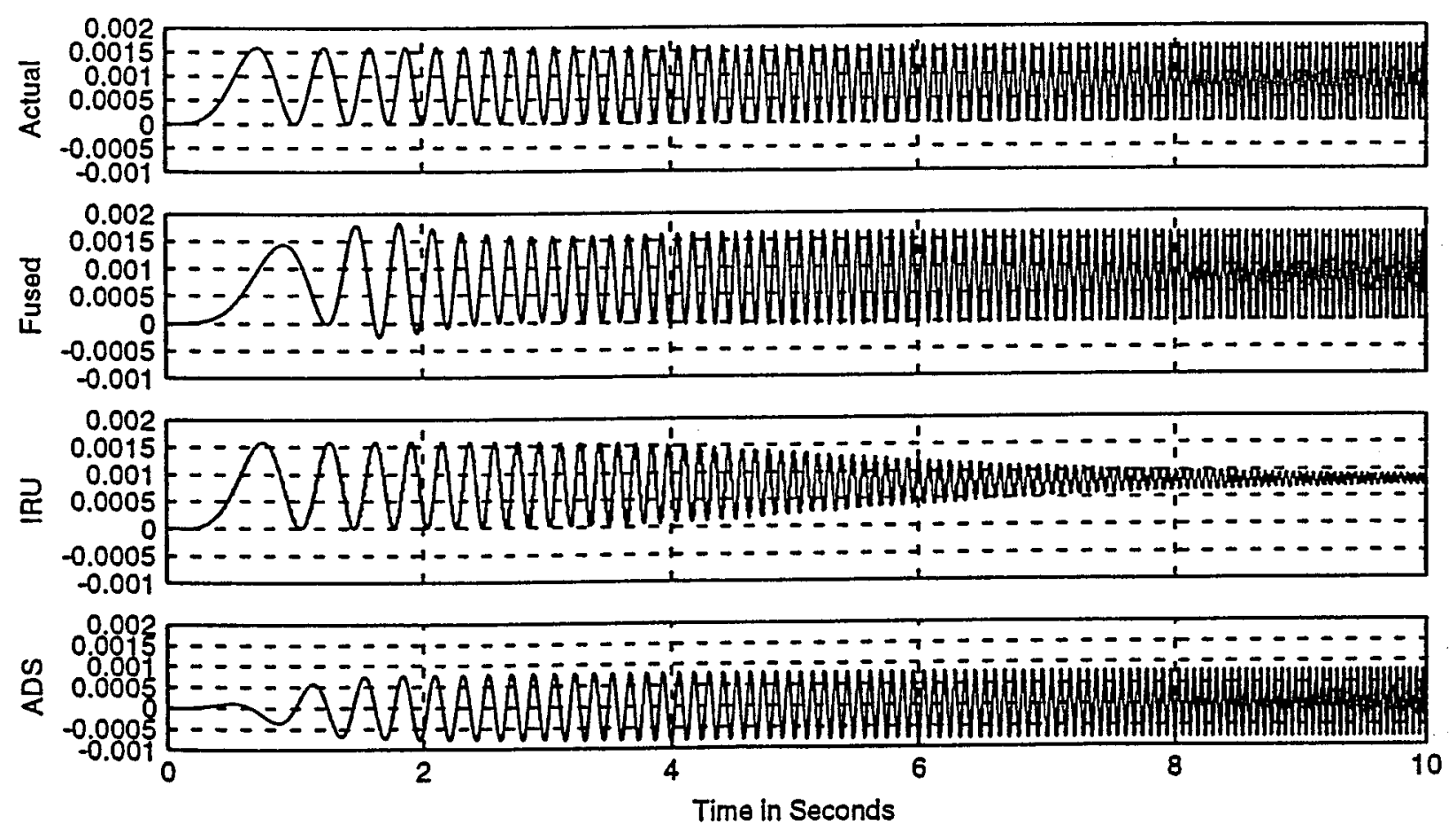

Fig. 6 Attitude angle corresponding to medium-rate sinusoidal frequency sweep (pitch axis).

tude angle generated using IRU measurements deteriorate significantly since the frequency content of this motion falls outside its bandwidth (see second trace from the bottom). Conversely, the ADS measurement contains the highfrequency data (see bottom trace), but lacks the near-dc component clearly present in the actual motion (see top and third traces). This is overcome by the ADS/IRU closed- loop sensor fusion method. Comparing the top two plots, it can be seen that the attitude angle produced by the ADS/ IRU fusion method reproduces the actual attitude angle with a high degree of fidelity, successfully blending the two types of measurements.

A second test case was developed to further demonstrate the capabilities of the closed-loop sensor fusion method.

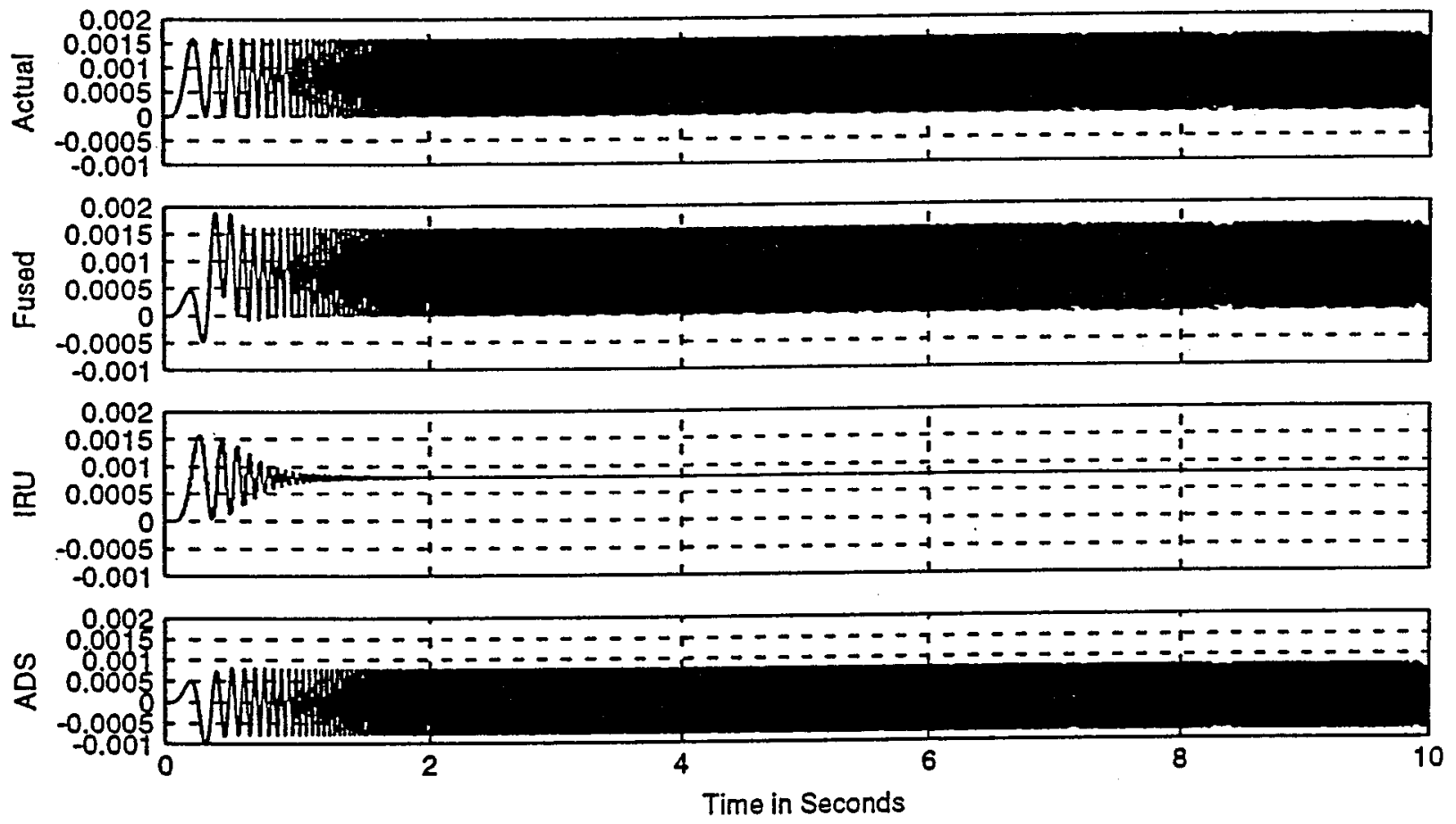

Fig. 7 Attitude angle corresponding to fast sinusoidal frequency sweep (yaw axis). 
Algrain: High-bandwidth attitude jitter determination ...

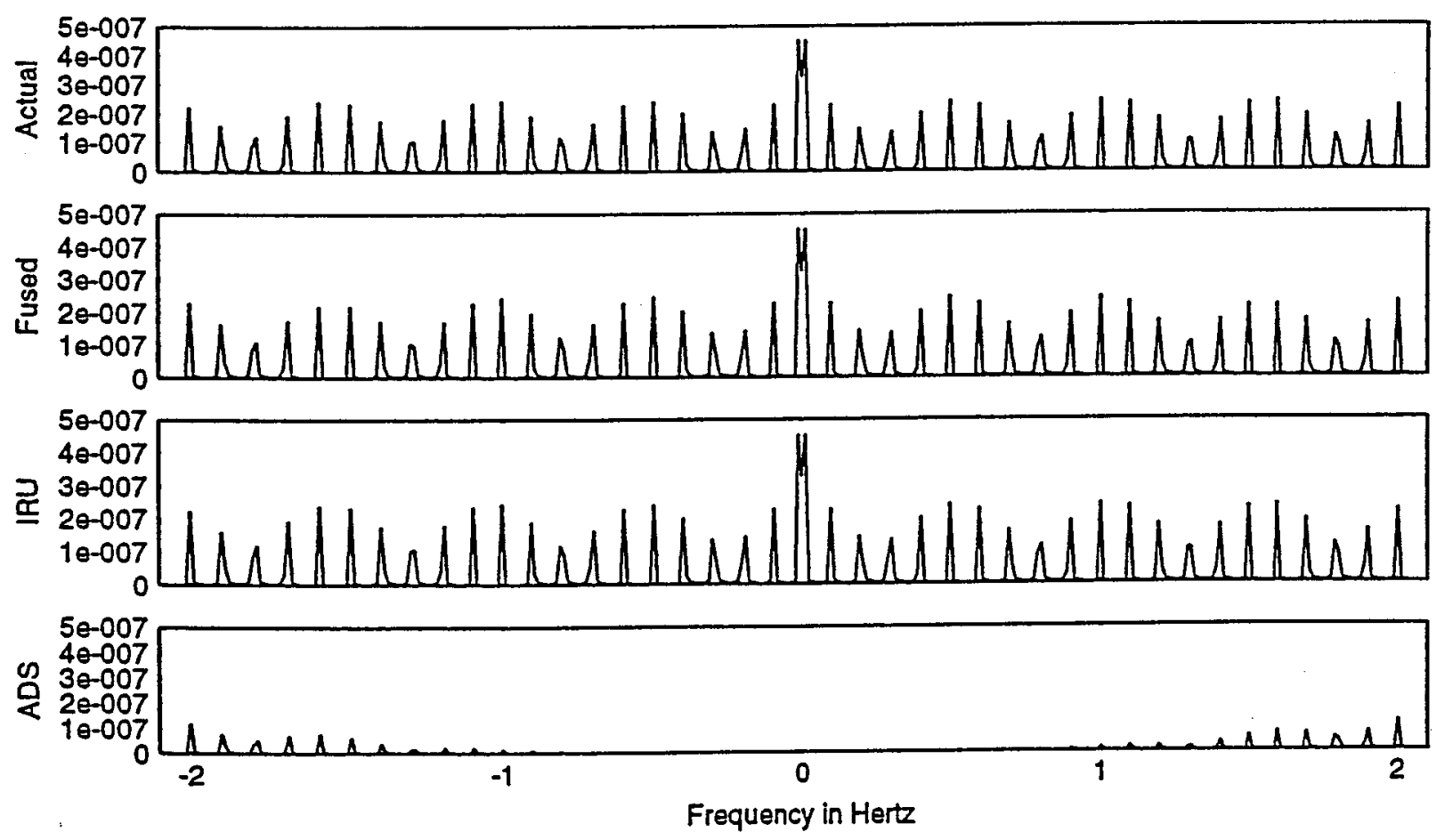

Fig. 8 Discrete power spectrum for low-frequency range in radians per squared seconds.

The motion is now composed of distinct sinusoidal tones (as opposed to a sinusoidal sweep). This facilitates the determination of frequency-response characteristics. The test scenario consists in extracting the attitude angles when the vehicle undergoes the following angular rates (in radians per second) along its axes:

Roll:

$$
\begin{aligned}
& \omega_{X}=10^{-3} \sum_{i} \omega_{i} \sin \left(\omega_{i} t\right) \\
& \text { with } \omega_{i}=0.2 \pi i t \text { and } i=1, \ldots, 20,
\end{aligned}
$$

Pitch: $\quad \omega_{Y}=10^{-3} \sum_{j} \omega_{j} \sin \left(\omega_{j} t\right)$

$$
\text { with } \omega_{j}=2.0 \pi j t \text { and } j=1, \ldots, 20 \text {, }
$$

Yaw:

$$
\begin{aligned}
\omega_{Z}=10^{-3} \sum_{k} \omega_{k} \sin \left(\omega_{k} t\right) \\
\quad \text { with } \omega_{k}=20 \pi k t \text { and } k=1, \ldots, 20 .
\end{aligned}
$$

Figure 8 shows (from top to bottom) the roll axis motion discrete power spectrums for actual, ADS/IRU fused, IRU, and ADS attitude angles. The frequency range of relevance extends from 0 to $2 \mathrm{~Hz}$. Within this frequency band, the ADS measurements contribute almost no information to the sensor fusion process, and the fused attitude angle relies almost exclusively on IRU measurements. This blending is quite effective in reproducing the actual motion as can be concluded from comparing the top two traces in Fig. 8.

The simulated results for the pitch axis motion are given in Fig. 9. The frequency content of this motion contains intermediate frequencies ranging from 0 to $20 \mathrm{~Hz}$. At these frequencies, there is considerable sensor bandwidth overlap (the ADS's starts at $2 \mathrm{~Hz}$ and the IRU's stops at $10 \mathrm{~Hz}$ ). This condition requires that the sensor fusion process blends the data from both sensors without gaining some frequencies more than others. After examining the plots in Fig. 9, it can be concluded that the ADS/IRU fusion process effectively combines the two measurements and provides good attitude data over the entire frequency range extending from 0 to $20 \mathrm{~Hz}$.

Finally, Fig. 10 shows the results corresponding to the fast motion along the yaw axis. In this case, the frequency range of interest spans from 0 to $200 \mathrm{~Hz}$. In contrast to the slow motion case (see Fig. 8), the sensor fusion now relies almost exclusively on the ADS measurements, with the exception of the dc term that is extracted from the IRU signal. Notice that the dc term is missing in the ADS spectrum (bottom trace), but present in the IRU spectrum (second trace from the bottom). In short, the spectrum of the actual attitude angle (top trace) and the ADS/IRU fused attitude angle (second trace from the top) show very good correspondence over the entire frequency range from 0 to 200 Hz. This demonstrates the effectiveness of the closed-loop control method for fusing low- and high-bandwidth angular measurements over a wide range of frequencies.

\section{Summary and Conclusions}

A new way of providing high-bandwidth attitude measurements is presented in this paper. The approach fuses measurements from sensors with different frequency response characteristics. This is needed because most sensors that provide good high-frequency data have poor near-dc response (high-pass characteristics), and those that provide good low-frequency information lack high-frequency sensi- 
Algrain: High-bandwidth attitude jitter determination ... .

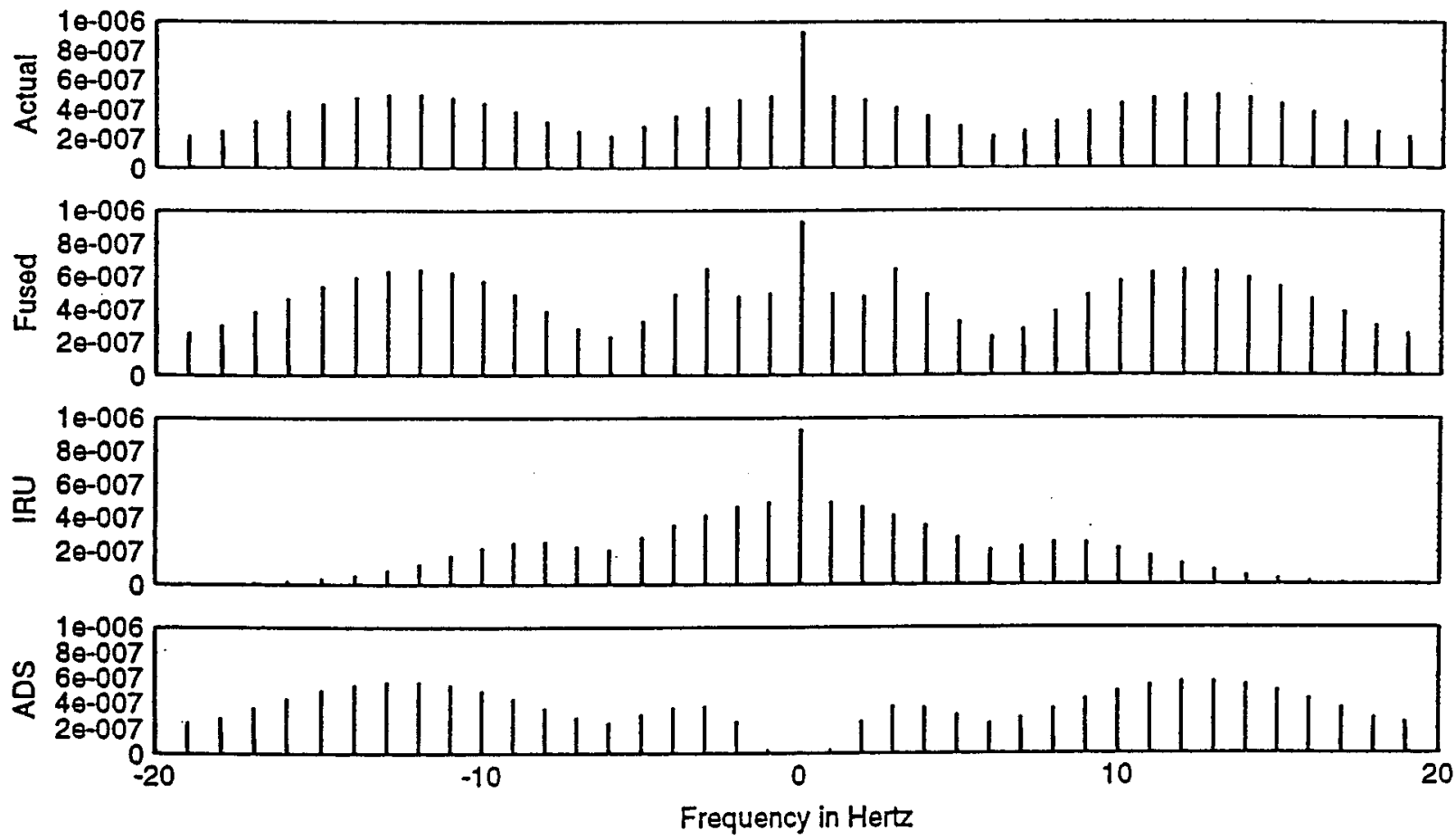

Fig. 9 Discrete power spectrum for midfrequency range in radians per squared seconds.

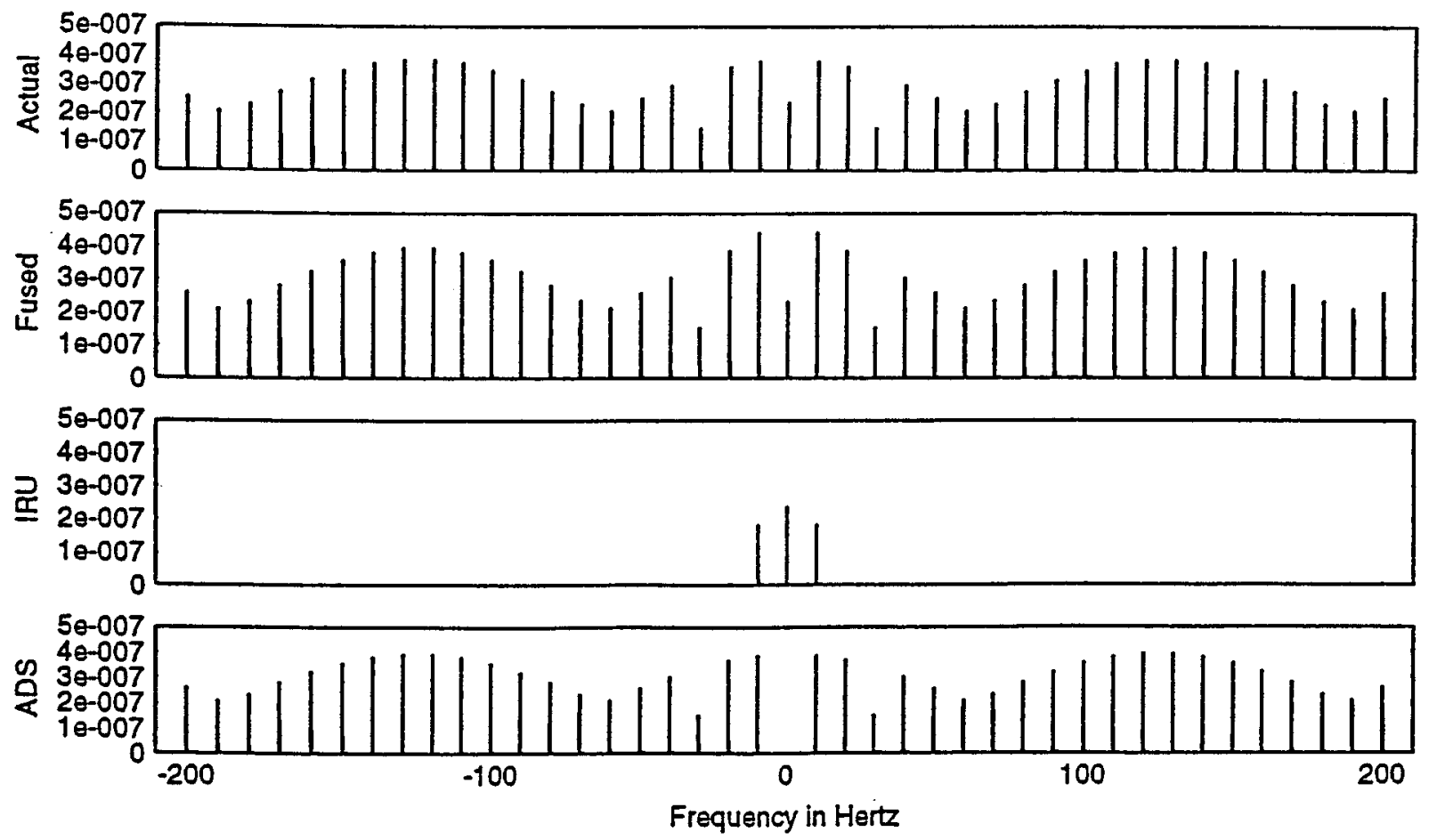

Fig. 10 Discrete power spectrum for high-frequency range in radians per squared second. 
tivity (low-pass characteristics). The data fusion is accomplished by incorporating a low-frequency correction term into the high-pass-type measurements. This correction is generated using a closed-loop control system that matches the long-term characteristics of attitude measurements obtained using high-pass sensors to those from low-pass sensors. A major advantage of this approach is that it does not rely on sensor models as traditional approaches, such as Kalman and complementary filtering, would. For clarity, the specific case of enhancing the bandwidth of an IRU using ADSs is presented. However, the methodology developed for this case applies to other sensor combinations as well. This is important since the algorithms would not have to be redeveloped if different sensors are used.

Although various control strategies are feasible, the structure selected uses a PID controller combined with an LPF. This approach is desirable because of its simplicity and effectiveness. To tune the controller, it was sufficient to try out few different gains in the simulation. Good performance was obtained after just a few trials, and the controller gains were varied significantly without losing stability. The performance of this closed-loop system is evaluated using a computer simulation. The attitude determination test consisted in resolving sinusoidal motion, along the three axes of rotation, with frequency contents in the range of 0 to $100 \mathrm{~Hz}$. The simulation confirmed that this approach is very effective in obtaining high-bandwidth attitude measurements. Finally, the simple PID-LPF combination could be replaced by a more sophisticated controller, such as one designed using $H_{\infty}$ methods, to further overcome sensor and other system uncertainties. Such enhancements would help achieve the full potential of this method, but the encouraging results obtained support the conclusion that this is a promising approach.

\section{Acknowledgments}

This work was sponsored in part by the National Aeronautics and Space Administration (NASA) Langley Research Center through the American Society for Engineering Education (ASEE) Summer Faculty Fellowship Program. We are especially thankful to Mr. Dave Cox for his valuable technical contributions, and to Dr. Douglas Price (Head of the Guidance and Controls Branch at Langley) for supporting this project.

\section{References}

1. R. Brown and P. Hwang "Applications and additional topics on discrete Kalman filtering,' Chap. 6, in Introduction to Random Signals and Applied Kalman Filtering, pp. 253-257, Wiley, New York, 1992.

2. J. Murrell, "Precision attitude determination for multimission spacecraft,', in Proc. AIAA Guidance and Control Conf., pp. 70-87 (1978).

3. E. Lefferts, F. Markley, and M. Shuster, "Kalman filtering for spacecraft attitude estimation,' J. Guid. (5), 417-429 (1982).

4. M. Algrain and R. Powers, "Line-of-sight pointing accuracy-stability analysis and computer simulation for small spacecraft," Proc. SPIE 2739, 62-76 (1996).

5. P. Hughes, "Rotational Kinematics,' Chap. 2, in Spacecraft Attitude Dynamics, pp. 22-31, Wiley, New York (1986).

6. P. Fortescue and J. Stark, "Space environment and its effects on spacecraft design,' Chap. 2 in Spacecraft Systems Engineering, pp. 56-57, Wiley, New York (1994); P. Fortescue and J. Stark, Chap. 10 in Spacecraft and Systems Engineering, pp. 244-245, Wiley, New York (1979).

7. H. Morris, "The inertial angular displacement sensor," DO \#87-15, Systron Donner Corp., Concord, CA (Feb. 1987).

8. D. Galler and A. Booth, "The shocking truth of accelerometer selection,' Mach. Design pp. 85-89 (July 6, 1989).

9. M. Algrain, "Gyroless line-of-sight stabilization for pointing and tracking systems,', Opt. Eng. 33(4), 1255-1260 (1994).

10. W. Press, B. Flannery, S. Teukolsky, and W. Vetterling, Numerical Recipes in C, Cambridge University Press, Cambridge, MA (1989).

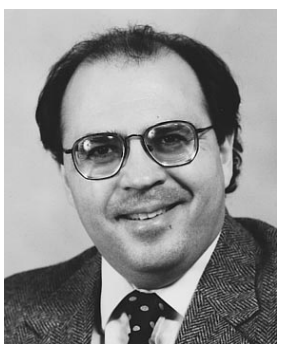

Marcelo C. Algrain received a BSME in 1980 and an MSEE in 1981, both from Wright State University, Dayton, Ohio, and a PhD in electrical engineering in 1991 from the Illinois Institute of Technology, Chicago. Since 1991 he has been an assistant professor at the University of Nebraska-Lincoln. From 1989 to 1991, he was an engineering specialist at Recon/ Optical, Inc., where he performed analyses on stabilization and control systems for high-resolution reconnaissance systems. From 1984 to 1989, he was with Borg-Warner Research, developing control system prototypes for a variety of automotive and industrial products. Prior to Borg-Warner, he was with Babcock \& Wilcox, designing process control systems for nuclear power plants. His research interests are stabilization, pointing, and tracking systems and stochastic, optimal, and adaptive controls, and he published numerous papers in the control systems field and holds two patents. Dr. Algrain is a member SPIE, IEEE, and AIAA. 